\title{
Synthesis of Mesoporous Zirconia by Using CTAB as Template
}

\author{
Salomón E. Borjas-García ${ }^{1}$, Ariosto Medina-Flores ${ }^{2}$, L. Béjar², C. Aguilar ${ }^{3}$, J. L. Bernal ${ }^{4}$. \\ ${ }^{1}$ Instituto de Física y Matematicas, Universidad Michoacana de San Nicolás de Hidalgo, Morelia, \\ Michoacán, México \\ ${ }^{2}$ Instituto de Investigaciones Metalúrgicas, Universidad Michoacana de San Nicolás de Hidalgo, \\ Morelia, Michoacán, México \\ ${ }^{3}$ Depertamento de Ingeniería Metalúrgia y Materiales. Universidad técnica Federico Santa María. \\ Valparaiso, Chile. \\ ${ }^{4}$ Automotive Mechanics Department. Universidad Politécnica de Pachuca. Zempoala, Hidalgo. México
}

A mesoporous material is a material which contains pores in a range between 2 and 50 nanometers [1]. This type of material can be used as catalyst for bulky reactant molecules due their large pore size. The first publication about synthesis of mesoporous materials by using a surfactant template was in 1992 by Mobil scientists [2]. After that, several methods have been develop for the synthesis of mesoporous metal oxides using cetyltrimethylammonium bromide (CTAB) $[1,3]$ or triblock copolymer non ionic surfactant as a template [1,4]. However, there is not enough research about the synthesis of mesoporous zirconia by using CTAB as template or the methods used are very complex. Mesoporous zirconia was synthesized using both Sol - Gel method with a hydrothermal soft treatment. The mesopores was prepared by using zirconium oxide chloride octahydrate (Sigma-Aldrich, purity 99.5\%), Sodium hydroxide (J.T.Baker, purity 97\%) and hexadecyltrimethyl ammonium bromide, CTAB (Sigma, purity 99\%) as source, alkaline material and template, respectively. In a first step, two solutions were prepared. The first one was obtained by dissolving $1.611 \mathrm{~g}$ of $\mathrm{ZrOCl}_{2} * 8 \mathrm{H}_{2} \mathrm{O}$ and 3.645 of CTAB in $20 \mathrm{~g}$ of distilled water. For the second solution, $0.4 \mathrm{~g}$ of $\mathrm{NaOH}$ was dissolved in $10 \mathrm{~g}$ of distilled water. In a second step, the Na-solution was added slowly (drop by drop) to Zr-solution and stirred. The final solution was stirred and heat in a hot stir plate at $90{ }^{\circ} \mathrm{C}$ to get a material with a molar ratio of $\mathrm{ZrOCl}_{2} * 8 \mathrm{H}_{2} \mathrm{O}: \mathrm{CTAB}: \mathrm{NaOH}: \mathrm{H}_{2} \mathrm{O}$ equal to $1: 2: 8: 75$. The gel obtained was aged in a polypropylene bottle at $80{ }^{\circ} \mathrm{C}$ for 1 day. After the hydrothermal treatment, the sample was washed with $100 \mathrm{ml}$ of distilled water and centrifuged at $4000 \mathrm{rpm}$. After that, the material was dried at $80{ }^{\circ} \mathrm{C}$ for 1 day. The powder X-ray diffraction patterns were collected with a siemens D5000 X-ray diffractometer equipped with graphite monochromatized high-intensity $\mathrm{CuK}_{\lambda}(\lambda=1.54178 \AA)$. The Bragg angle $2 \theta$ ranges from $1^{\circ}$ to $8^{\circ}$ at a scanning rate of $0.02^{\circ} / 0.6 \mathrm{~s}$. The surface morphology images of the samples were analyzed by using a scanning electron microscopy FEG-SEM JEOL JSM 7600. Figure 1 shows XRD pattern of zirconia synthesized. This figure shows a characteristic peak about the presence of mesopores in the material around 1.04 degrees. Figure 2a shows an image of zirconia material which has mesopores with a size around $28 \mathrm{~nm}$. Figure $2 \mathrm{~b}$ shows the EDS spectrum taken over material analyzed. The results showed that the molar relation between template and zirconium could be crucial in the formation of mesopores. However, by soft hydrothermal treatment and low amount of water could help the formation of mesoporos in the material let us developed an easy procedure for the synthesis, which can be applied at different materials. 


\section{References}

[1] D.W. Bruce, D. O’Hare and R.I. Walton, "Porous materials", 1st ed. (Wiley, United Kingdom, 2010) p. 1.

[2] C. T. Kresge et al, Nature Volume 359 (1992) p. 710.

[3] Z. R. Tian et al, Science Volume 276 (1997) p. 926.

[4] P. Yang et al, Nature Volume 396 (1998) p. 152.

[5] The authors acknowledge funding from H. Consejo Técnico of Institute of Physics and Mathematics, and Consejo Nacional de Ciencia y Tecnología (CONACyT), México.

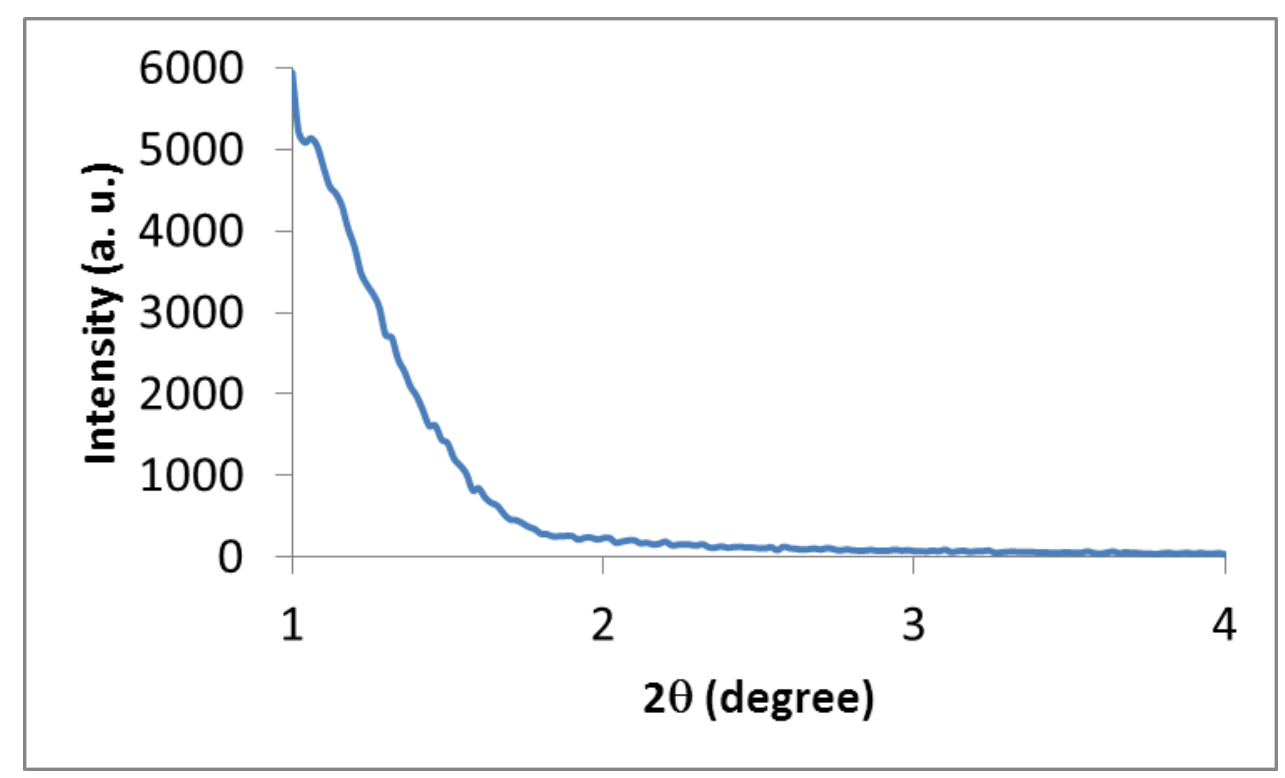

Figure 1. XRD pattern of mesoporous zirconia.
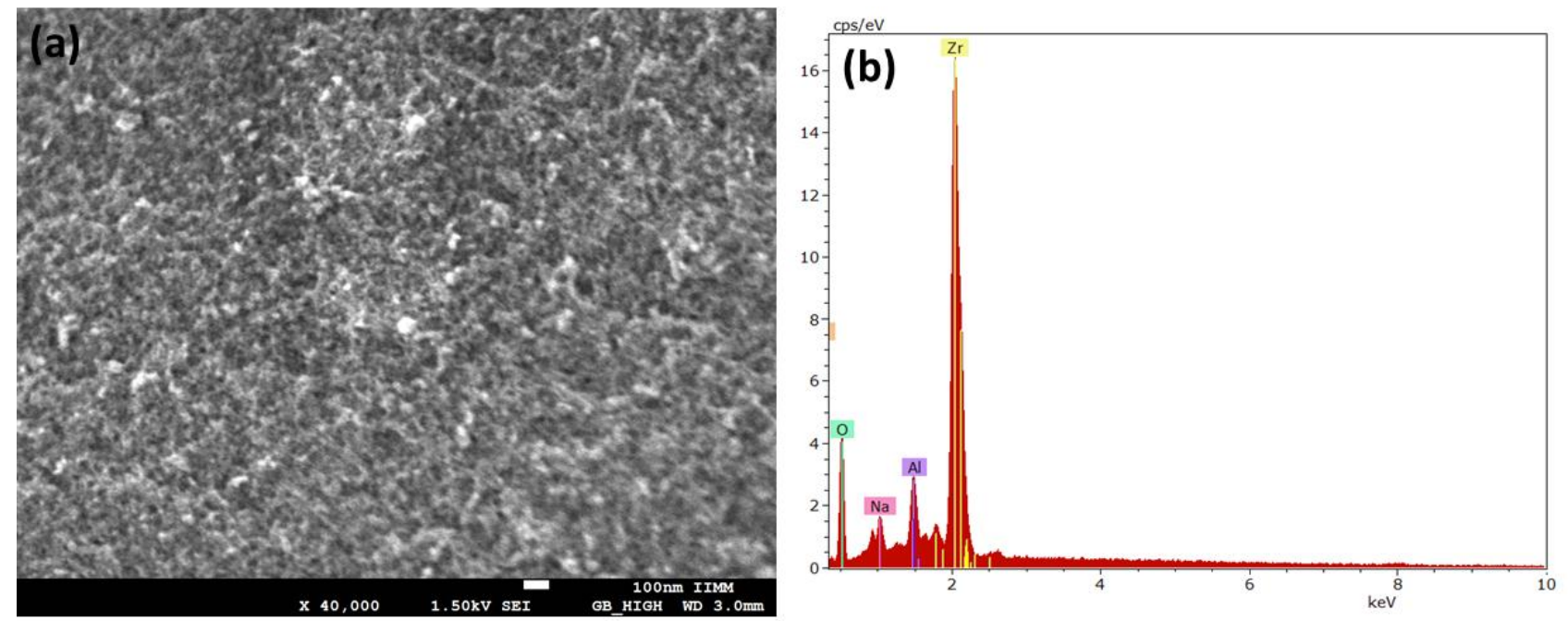

Figure 2. a) SEM image of mesoporous zirconia after synthesis (40000x of magnification), b) EDS spectrum taken from over one particle. 